\title{
The Relationship Between Sonographic Findings, and Clinical and Paraclinical Symptoms in Henoch Schonlein Purpura
}

\author{
Houman Alizadeh, ${ }^{1}$ Mojtaba Ranjbar, ${ }^{1}$ Vahid Ziaee, ${ }^{2,3,}{ }^{*}$ Reihaneh Mohsenipour,,4 Mehrzad \\ Mehdizadeh, ${ }^{1,5}$ Mohammad-Hassan Moradinejad, ${ }^{1,3}$ Mozhgan Sabbaghian, ${ }^{3}$ and Hossein Alimadadi ${ }^{1,3}$ \\ ${ }^{1}$ Children's Medical Center, Pediatric Center of Excellence, Tehran, Iran \\ ${ }^{2}$ Pediatric Rheumatology Research Group, Rheumatology Research Center, Tehran University of Medical Sciences, Tehran, Iran \\ ${ }^{3}$ Department of Pediatrics, Tehran University of Medical Sciences, Tehran, Iran \\ ${ }^{4}$ Growth and Development Research Center, Tehran University of Medical Sciences, Tehran, Iran \\ ${ }^{5}$ Department of Radiology, Tehran University of Medical Sciences, Tehran, Iran \\ "Corresponding author: Vahid Ziaee, MD, Division of Pediatric Rheumatology, Children's Medical Center, No. 62 Dr. Gharib St., Keshavarz Blvd, Tehran 14194, IR Iran. E-mail: \\ ziaee@tums.ac.ir
}

Received 2016 April 20; Revised 2016 October 14; Accepted 2016 November 21.

\section{Abstract}

Background: Gastrointestinal (GI) manifestations are common in patients with Henoch Schonlein Purpura (HSP) and it seems that ultrasound is the first modality for detecting GI involvement. This study was performed to evaluate the relationship between sonographic findings and clinical, and paraclinical symptoms.

Methods: All patients with HSP referred to our clinic in 2011 and 2012, were enrolled in the study. The data including sonographic and other lab tests were collected and analyzed, and the association between sonographic findings and clinical, and paraclinical symptoms were evaluated.

Results: Among 112 patients (68 males and 44 females), 28 cases had abnormality in their sonography that was higher in patients with GI and renal symptoms. Furthermore, length of hospitalization and need for corticosteroids was greater in patients with positive sonographic findings.

Conclusions: In patients with HSP, ultrasound is a valuable modality to determine the prognosis of the disease.

Keywords: Henoch Schonlein Purpura, Ultrasound, Gastrointestinal Involvement, Vasculitis, Abdominal Pain, Children

\section{Background}

Henoch Schonlein Purpura (HSP) is the most common vasculitis in children (1-3) and is characterized by nonthrombocytopenic purpura, abdominal pain, skin rash, arthritis and arthralgia, genital, and renal involvement (4, 5). Although, its etiology is unknown, history of previous infection, vaccination, drug exposure, and certain kinds of food have been determined in various cases (4-7).

Gastrointestinal (GI) manifestation can be found in two thirds of cases. It commonly occurs after skin rash, yet, can appear before other symptoms and causes unnecessary laparotomy or testicular involvement $(4,5)$. Gastrointestinal symptoms include abdominal pain, nausea, vomiting, diarrhea, bleeding, intussusception, and mesenteric ischemia or perforation (2). Ultrasound (US) is an effective method of choice for screening gastrointestinal symptoms in children (8).

Although radiographic study and US demonstrate abnormal results in many cases, our knowledge about the association between sonographic results and clinical symptoms, and prognosis is not sufficient. This study was there- fore undertaken to assess the efficacy of US in diagnosis of GI involvement of HSP and the relationship of these findings with clinical and paraclinical symptoms, course of disease, its complications, and length of hospitalization.

\section{Methods}

This study was approved by the ethics committee of Tehran University of Medical Sciences. All patients with HSP, who were referred from 2011 to 2012 to the Children's Medical Center, a tertiary pediatric center in Tehran, were enrolled in this study. Indication for admission was active phase of disease (first week after disease onset), and/or after first week with active skin lesion, and organ involvement. Diagnosis of HSP was confirmed based on International League of Associations for Rheumatology (ILAR) criteria for HSP (9). If diagnosis was disproved during evaluation and/or patients had concurrent other chronic disease, they were excluded from the study.

A series of laboratory tests including complete blood cell, erythrocyte sedimentation rate, C-reactive protein, 
urine analysis (U/A), and stool exam (S/E) followed complete physical examination. Urine analysis and $\mathrm{S} / \mathrm{E}$ were repeated daily in each patient during the hospital stay. Abdominal sonography was performed in all patients with and without GI involvement during 48 hours after admission. The US apparatus used in this study was Siemens G50 with a linear probe 5-10 MHZ. Bowel wall thickening more than $2 \mathrm{~mm}$ was considered significant. Small bowel diameter was measured by 2 radiologists. Enteral wall thickening (small bowel diameter more than $4 \mathrm{~mm}$ ) was taken for as a prognostic factor. All patients with GI involvement were treated with prednisolone. Based on US findings, patients were divided to 2 groups; group A with abnormal GI in US and group B with normal US.

Statistical Package for social sciences, version 17.0 was used for statistical analysis. Statistical significance was set at 0.05. Man-Whitney test and Fisher exact test were used to compare the data.

\section{Results}

Overall, 112 patients (61\% males and 39\% females, average age of 6 , range 2 to 16 years) were enrolled in this study. Male to female ratio was $1.5: 1$ and $78 \%$ of patients were 3 to 10 years old. Sonographic evaluations of 28 (25\%) cases showed abnormalities (group A) including bowel wall thickening, intussusception, and disruption of bowel wall differentiation. Other patients (group B) had normal sonographic findings.

Three cases of group A underwent surgery because of acute abdomen. Fifty percent of group A had one recurrence in the long term follow up; this was $4 \%$ for group $B$.

Duration of hospitalization was significantly higher for group A (16 days versus 7 days for group $B)(P<0.0001)$. Involvement of different organs in the two groups is summarized in Table 1. Renal involvement had a positive association with GI involvement in US evaluation and it was significantly higher for group $\mathrm{A}(\mathrm{P}<0.0001)$.

Table 1. The Involvement of Organs in Two Groups of Patients with Henoch Schonlein Purpura ${ }^{\mathrm{a}}$

\begin{tabular}{lccc}
\hline Involvement & Group A, (n=28) & Group B, (n=84) & P Value \\
\hline Skin & $28(100)$ & $84(100)$ & \\
Gastro-intestinal & $27(96)$ & $59(70)$ & 0.01 \\
Joint & $25(89)$ & $63(75)$ & 0.15 \\
Renal & $18(64)$ & $16(19)$ & 0.0001 \\
\hline Testicle (in male) & $5(33)$ & $15(28)$ & 0.5 \\
\hline
\end{tabular}

${ }^{\mathrm{a}}$ Value are expressed as No. (\%).
Table 2 shows the results of lab tests. There was an association between US findings and microscopic or macroscopic lower GI bleeding. Positive C-Reactive Protein (CRP) and anemia were significantly more common for group A.

Table 2. The Results of Laboratory Tests in Patients with Henoch Schonlein Purpura ${ }^{a}$

\begin{tabular}{|c|c|c|c|}
\hline Variable & $\operatorname{Group} A(n=28)$ & Group $B(n=84)$ & P Value \\
\hline $\begin{array}{l}\text { Positive stool exam } \\
\text { (occult blood/RBC) }\end{array}$ & $21(75)$ & $8(10)$ & 0.0001 \\
\hline Thrombocytosis & $9(32)$ & $21(25)$ & 0.3 \\
\hline Leukocytosis & $19(68)$ & $57(68)$ & 1 \\
\hline Anemia & $19(68)$ & $35(42)$ & 0.005 \\
\hline Positive CRP & $25(89)$ & $55(65)$ & 0.02 \\
\hline Increased IgA & $11(39)$ & $27(32)$ & 0.3 \\
\hline Increased IgM & 0 & $3(4)$ & 0.02 \\
\hline Increased IgG & $5(18)$ & $12(14)$ & 0.4 \\
\hline Positive ANA & $2(7 \%)$ & $5(6)$ & 0.8 \\
\hline Increased ASO titer & $3(11)$ & $16(19)$ & 0.1 \\
\hline
\end{tabular}

Sixty-four percent of patients (27 (96\%) in group A and 45 (54\%) in group B) were treated with corticosteroids ( $\mathrm{P} \leq$ $0.0001)$.

Five (17.8\%) patients in group A underwent operation for intussusception, in 3 of whom HSP rash appeared after surgery. Six (5\%) patients in group B had severe glomerulonephritis, which required immunosuppressive therapy, and among these cases, 5 were in group $A$ and 1 in group $B$ $(\mathrm{P} \leq 0.0001)$. Positive predictive value of US for diagnosis of GI involvement and positive blood in stool examination were $58 \%$ and $88 \%$, respectively.

\section{Discussion}

This study aimed to evaluate the possibility of predicting GI involvement in clinical course, complications and duration of hospitalization of HSP patients by ultrasound. We found GI symptoms in $77 \%$ of our patients whereas a range of 51 to $78 \%$ was reported in other studies (10-12). The average duration of hospitalization in patients with GI involvement was higher than without it $(12,13)$. The US study can be used as the modality of choice for evaluation of GI involvement (12-16). Similar to our study, duration of hospitalization in patients with abnormal sonographic findings was significantly higher than that in patients with normal sonographic evaluation in Nchimi's study (13). There was 
a positive association between GI symptoms and positive sonographic findings that were significantly different in the group with normal US findings. In this group, 96\% of the patients required corticosteroid therapy similar to other studies $(12,13)$.

In this study, the overall recurrence of abdominal symptoms during the 1-month follow up was $16 \%$, which was similar to Chen's study (12). Similar to Nchimi's study (13), this rate was higher in patients with abnormal sonographic findings (50\% versus $4 \%$ with normal US). In our study, $2.5 \%$ of patients had gastrointestinal symptoms (including intussusception) before manifestation of skin rash. Other authors have reported this as $10 \%$ to $17 \%(10,12$, 17).

Evidence of GI bleeding had a significant statistical difference in the group with and without abnormal GI sonographic findings. Although, in previous reports a range of $18 \%$ to $52 \%$ of patients with HSP had occult or overt bleeding $(10,12)$, this association between sonographic findings and GI bleeding was not reported in other studies. Prediction value of GI involvement by US was $58 \%$ in this study, which is less than that reported by Nchimi et al. (13). This value for prediction of GI bleeding in stool examination was $88 \%$.

In our study, $78 \%$ of patients were 3 to 10 years old, which was fairly similar to other published data $(2,10$, 15). All of our patients developed skin palpable purpura as mentioned in a previous article, although it maybe not the first manifestation of $\operatorname{HSP}(10-12,17)$. Joint involvement was seen in $79 \%$ of patients and a range of $43 \%$ to $82 \%$ was reported in other studies $(10,11,17)$.

Renal involvement (hematuria and proteinuria) was observed in $30 \%$ of our patients and in other studies a wide range (32\% to $54 \%$ ) was reported $(10,17)$. Renal involvement was fairly higher in patients with positive GI sonographic findings in proportion to negative GI sonographic findings with a significant statistical difference.

In this study, the rate of anemia was different in the two groups of patients. This can be due to extra volume of blood excretion from stool or intramural bleeding in patients with positive sonographic findings.

Positive CRP was significantly higher in the group with positive GI sonographic findings. It may be a better factor for determining patients' prognosis in proportion to other acute phase reactants such as Erythrocyte Sedimentation Rate (ESR).

\subsection{Conclusion}

In conclusion, US is the imaging modality of choice for determining HSP prognosis in patients as there are various types of bowel wall US findings in children with HSP that have an association with clinical symptoms such as renal and GI manifestation. Ultrasound can predict GI involvement before manifestation of symptoms, prognosis of HSP, and duration of hospitalization.

\subsection{Limitation}

The retrospective nature of this study caused a lack of exact data in some medical folders.

\section{Acknowledgments}

This study was financially supported by Tehran University of Medical Sciences.

\section{Footnote}

Conflict of Interest: The authors declare that they had no conflicts of interest

\section{References}

1. Ballinger S. Henoch-Schonlein purpura. Curr Opin Rheumatol. 2003,15(5):591-4. doi: 10.1097/00002281-200309000-00012. [PubMed: 12960486].

2. Eleftheriou D, Brogan PA. Vasculitis in children. Best Pract Res Clin Rheumatol. 2009;23(3):309-23. doi: 10.1016/j.berh.2009.02.001. [PubMed: 19508940].

3. Ebert EC. Gastrointestinal manifestations of Henoch-Schonlein Purpura. Dig Dis Sci. 2008;53(8):2011-9. doi: 10.1007/s10620-007-0147-0. [PubMed: 18351468].

4. Masuda M, Nakanishi K, Yoshizawa N, Iijima K, Yoshikawa N. Group A streptococcal antigen in the glomeruli of children with Henoch-Schonlein nephritis. Am J Kidney Dis. 2003;41(2):366-70. doi: 10.1053/ajkd.2003.50045. [PubMed:12552498].

5. Paydary K, Emamzadeh Fard S, Mahboubi AH, Ziaee V, Moradinejad MH, Kajbafzadeh AM. Penile Skin Involvement as the First Presentation of Henoch-Schonlein Purpura Report of Nine Cases and Review of Literature. Iran J Pediatr. 2015;25(4):2177. doi: 10.5812/ijp.2177. [PubMed: 26396696].

6. Lambert EM, Liebling A, Glusac E, Antaya RJ. Henoch-Schonlein purpura following a meningococcal vaccine. Pediatrics. 2003;112(6 Pt 1):491. doi: 10.1542/peds.112.6.e491. [PubMed: 14654652].

7. Kalman S, Ibrahim Aydin H, Atay A. Henoch-Schonlein purpura in a child following varicella. J Trop Pediatr. 2005;51(4):240-1. doi: 10.1093/tropej/fmi065. [PubMed: 15980021].

8. Vasavada P. Ultrasound evaluation of acute abdominal emergencies in infants and children. Radiol Clin North Am. 2004;42(2):445-56. doi: 10.1016/j.rcl.2004.01.003. [PubMed:15136027].

9. Ozen S, Pistorio A, Iusan SM, Bakkaloglu A, Herlin T, Brik R, et al. EULAR/PRINTO/PRES criteria for Henoch-Schonlein purpura, childhood polyarteritis nodosa, childhood Wegener granulomatosis and childhood Takayasu arteritis: Ankara 2008. Part II: Final classification criteria. Ann Rheum Dis. 2010;69(5):798-806. doi: 10.1136/ard.2009.116657. [PubMed: 20413568].

10. CalviÑO MC, Llorca J, GarcíA-PorrÚA C, FernÁNdez-Iglesias JL, Rodriguez-Ledo P, GonzÁLez-Gay MA. Henoch-schönlein purpura in children from northwestern spain. Medicine. 2001;80(5):279-90. doi: 10.1097/00005792-200109000-00001. 
11. Chang WL, Yang YH, Lin YT, Chiang BL. Gastrointestinal manifestations in Henoch-Schönlein purpura: a review of 261 patients. Acta Paediatr. 2007;93(11):1427-31. doi:10.1111/j.1651-2227.2004.tb02623.x.

12. Chen SY, Kong MS. Gastrointestinal manifestations and complications of Henoch-Schonlein purpura. Chang Gung Med J. 2004;27(3):175-81. [PubMed: 15148994].

13. Nchimi A, Khamis J, Paquot I, Bury F, Magotteaux P. Significance of bowel wall abnormalities at ultrasound in Henoch-Schonlein purpura. J Pediatr Gastroenterol Nutr. 2008;46(1):48-53. doi: 10.1097/01.mpg.0000304453.99799.8c. [PubMed: 18162833].

14. Özdemir H, Işik S, Buyan N, Hasanoglu E. Sonographic demonstration of intestinal involvement in Henoch-Schönlein syndrome. Eur J Radiol. 1995;20(1):32-4. doi:10.1016/0720-048x(95)00609-t.
15. Kagimoto S. Duodenal findings on ultrasound in children with Schonlein-Henoch purpura and gastrointestinal symptoms. $J$ Pediatr Gastroenterol Nutr. 1993;16(2):178-82. doi: 10.1097/00005176199302000-00014. [PubMed: 8450386].

16. Connolly B, O'Halpin D. Sonographic evaluation of the abdomen in henoch-schonlein purpura. Clin Radiol. 1994;49(5):320-3. doi: 10.1016/s0009-9260(05)81796-9.

17. Trapani S, Micheli A, Grisolia F, Resti M, Chiappini E, Falcini F, et al. Henoch Schonlein purpura in childhood: epidemiological and clinical analysis of 150 cases over a 5-year period and review of literature. Semin Arthritis Rheum. 2005;35(3):143-53. doi: 10.1016/j.semarthrit.2005.08.007. [PubMed: 16325655]. 